\title{
PREPARAÇ̃̃o DE MATERIAL A BASE DE FERRO/CARVÃO A PARTIR DE RESÍDUO DO BIODIESEL PARA APLICAÇÃO NA REMOÇÃO DE CONTAMINANTES ORGÂNICOS
}

\author{
Rochele Ferreira Silva Diniz ${ }^{\mathrm{a}}$, Thais Aline Prado Mendonça ${ }^{\mathrm{a}}$, Raquel Aparecida Domingues ${ }^{\mathrm{a}, \sharp} \mathbf{e}_{\text {Maraísa Gonçalves }}{ }^{\mathrm{a}, *,(\mathbb{D})}$ \\ a'Universidade Federal de São Paulo, Instituto de Ciência e Tecnologia, Rua Talim, 330, 12231-280 São José dos Campos - SP, Brasil
}

Recebido em 09/12/2020; aceito em 11/02/2021; publicado na web em 18/03/2021

\begin{abstract}
PREPARATION OF IRON/CARBON MATERIAL FROM BIODIESEL WASTE FOR APPLICATION IN THE REMOVAL OF ORGANIC CONTAMINANTS. In this work, iron/carbon-based catalysts obtained from biodiesel residues, glycerin, were prepared. The composites were prepared by thermal carbonization of the glycerin polymer, from biodiesel residues, in the presence of iron salt. The materials obtained were characterized by adsorption/desorption of $\mathrm{N}_{2}$, scanning electron microscopy (SEM), X-ray diffraction (XRD), Raman spectroscopy and thermogravimetric analysis. XRD analysis have shown the presence of hematite and magnetite. The methylene blue (MB) adsorption and oxidation tests reached $99 \%$ color removal in the presence of hydrogen peroxide. The steadystate spectra confirmed the MB oxidation by the reduction of the fluorescence intensity. Besides, it has high stability, with no active phase leaching, proving a heterogeneous oxidation process.
\end{abstract}

Keywords: materials, iron/carbon; biodiesel waste; heterogeneous oxidation.

\section{INTRODUÇÃO}

A poluição ambiental do solo, ar e água intensificou de forma considerável nas últimas décadas, motivando discussões sobre formas de diminuir os impactos causados ao meio ambiente e à sociedade. Uma preocupação atual é a queima de combustíveis fósseis, ocasionando poluição do ar e queima de matéria-prima nobre, sendo necessárias políticas públicas para incentivo da utilização de biocombustíveis. ${ }^{1}$

O biodiesel é um biocombustível obtido de óleos vegetais, bem como de gorduras animais. Os óleos podem ser extraídos para este fim ou mesmo pelo reaproveitamento de óleos usados. O processo de obtenção de biodiesel ocorre via reação de transesterificação dos triglicerídeos em presença de álcool e um catalisador. Porém, além do biodiesel, é produzido um subproduto, a glicerina bruta, a qual representa $10 \%$ da produção total. ${ }^{1-3}$

No Brasil, atualmente, é obrigatória a utilização de $10 \%$ de biodiesel ao diesel de petróleo (B10) e tem-se como meta a implementação da mistura de $15 \%$ até 2023 . $^{4,5}$

Com isso, é iminente a elevação da produção de biodiesel e, consequentemente, a geração de um excesso de resíduo. No ano de 2019, foram produzidos aproximadamente $590.110 \mathrm{~m}^{3}$ de glicerina bruta. ${ }^{4}$ A glicerina bruta é uma mistura de 70 a $80 \%$ de glicerol e outros componentes como álcool, catalisador, reagentes, entre outras impurezas. ${ }^{1}$ Atualmente, várias pesquisas têm sido realizadas para propor novas rotas de transformação da glicerina, visto que a maioria dos processos industriais que utiliza glicerol como matéria-prima, o faz em sua forma pura. A purificação da glicerina bruta possui custo elevado, o que inviabiliza sua utilização como fonte de glicerol para uso industrial. ${ }^{1,2}$ Com isso, fazem-se necessárias mais pesquisas para proposição de novas tecnologias com intuito de agregar valor a este subproduto do biodiesel, a glicerina.

A glicerina bruta é composta de moléculas que, basicamente, possuem em sua estrutura carbono, hidrogênio e oxigênio. Assim, uma das possibilidades para o aproveitamento deste resíduo é a produção de materiais carbonáceos, tais como carvões ácidos, carvão ativado,

*e-mail: goncalves.maraisa@unifesp.br

\#e-mail: radomingues@unifesp.br materiais metal/carvão, entre outros. ${ }^{1,2,6} \mathrm{Na}$ literatura, são relatadas pesquisas para utilização de glicerol na preparação de materiais à base de carbono, descrevendo como grande vantagem a baixa presença ou mesmo ausência de componentes inorgânicos. ${ }^{7}$ Com isso, diversos autores aproveitam esta grande vantagem para preparação de catalisadores ácidos e carvões ativados para aplicações diversas. ${ }^{8-13}$ Os pioneiros na preparação de carvão ácido a partir de glicerol foram Prabhavathi Devi e colaboradores, utilizando uma proporção de 1:4, glicerol:ácido, obtendo um material com elevada atividade catalítica. ${ }^{11}$ Posteriormente, Prabhavathi Devi e colaboradores também prepararam outros carvões ácidos para utilização como catalisador em diferentes reações, obtendo uma boa conversão. ${ }^{10,14}$ Assim, os autores evidenciaram a viabilidade de polimerização de glicerol para preparação de materiais carbonáceos..$^{10,14}$ Gonçalves e colaboradores estudaram a preparação de carvão ácido a partir de glicerina bruta obtendo elevada acidez superficial e alta estabilidade como catalisador em reações de conversão de eterificação do glicerol e em conversão de frutose. ${ }^{8,9}$ Porém, na literatura não há relato de utilização de resíduo de glicerina bruta para preparação de material a base ferro/carvão pelo método in situ: impregnação de ferro simultâneo à polimerização e carbonização, sendo a inovação deste trabalho.

Outro problema atual é a poluição aquática devido aos contaminantes orgânicos diversos, alguns com toxicidade aguda, persistência e natureza cancerígena. A presença desses contaminantes em cursos de água é causada pelo consumo e descarte de diversos produtos tais como de higiene pessoal, indústria farmacêutica, indústria têxtil e química. Os efluentes da indústria têxtil tem despertado preocupação devido à grande variedade e recaucitrância de alguns compostos, causando grande impacto na vida aquática. ${ }^{15}$ As técnicas mais usuais para a remoção de contaminantes orgânicos são tratamentos biológicos e físico-químicos. Para alguns efluentes industriais, o tratamento biológico não é eficiente devido à presença de poluentes estáveis e tóxicos. ${ }^{16}$ Assim, a remoção desses contaminantes pelo processo de adsorção ou oxidação faz-se necessária devido a não seletividade desses processos. A adsorção é um processo no qual o adsorbato interage com o adsorvente por interações físico-químicas. A oxidação de contaminantes orgânicos em meio aquoso via processos oxidativos avançados tais como oxidação eletroquímica, fotocatálise, ozonização, foto-Fenton, Fenton, entre outros, envolve a geração de 
radicais altamente oxidantes e por isso tem ganhado destaque. ${ }^{17,18} \mathrm{O}$ processo Fenton envolve a mistura homogênea em solução aquosa contento ferro iônico $\left(\mathrm{Fe}^{+2}\right.$ ou $\left.\mathrm{Fe}^{+3}\right)$ e peróxido de hidrogênio, resultando na formação de radicais oxidantes como o hidroxila $\left({ }^{\circ} \mathrm{OH}\right)$ ou hidroperóxido $\left({ }^{\circ} \mathrm{OOH}\right) .{ }^{19}$ Devido ao mecanismo radicalar da reação, ela apresenta grande eficiência para tratamento de vários efluentes nos quais estão presentes diferentes contaminantes orgânicos, ocorrendo a degradação por processos complexos, envolvendo várias reações. Geralmente, esses processos podem ser agrupados em reações de iniciação, propagação e terminação. ${ }^{20}$ $\mathrm{O}$ processo Fenton homogêneo só possui eficiência em meio ácido, pH 2 a 4, e com concentração de ferro de 40 a $80 \mathrm{mg} \mathrm{L}^{-1}$, necessitando tratamento posterior para precipitação do ferro solúvel com intuito de alcançar níveis previstos pela legislação vigente para posterior descarte de efluente, $15 \mathrm{mg} \mathrm{L}^{-1} \cdot{ }^{21}$ Com intuito de eliminar essas desvantagens, a utilização de um catalisador sólido à base de ferro tem ganhado destaque, o processo Fenton heterogêneo. $\mathrm{Na}$ literatura, é relatada por diversos autores a utilização de ferro disperso em carvão ativado, sílica, zeólita ou mesmo ferro puro para tratamento de inúmeros contaminantes. ${ }^{22-26}$

Diante disso, neste trabalho foi proposta a produção de material à base de ferro/carvão a partir de resíduos de biodiesel (glicerina bruta) para aplicação em remoção de contaminantes orgânicos em meio aquoso. $\mathrm{O}$ catalisador obtido neste trabalho foi preparado por um método in situ, sem necessidade de obtenção do carvão e posterior impregnação, o que representa um novo processo para transformação do resíduo do biodiesel. A atividade catalítica dos materiais preparados foi estudada na remoção de azul de metileno via espectroscopia de UV-visível e emissão de fluorescência.

\section{PARTE EXPERIMENTAL}

\section{Preparação do material ferro/carvão}

A preparação dos materiais foi realizada em duas etapas. $\mathrm{Na}$ primeira etapa, ocorreu a impregnação/polimerização simultânea da glicerina bruta com intuito de obter o polímero com ferro incorporado em sua matriz. Na segunda etapa, ocorreu o processo de tratamento térmico em atmosfera inerte para formação de um material carbonáceo estável, o material à base de ferro/carvão.

A impregnação/polimerização da glicerina bruta (Oxiteno) foi realizada à temperatura de $180^{\circ} \mathrm{C}$, por $3 \mathrm{~h}$ sob agitação constante. Foram misturados 22,50 g de glicerina bruta, 2,20 g de ácido sulfúrico (Vetec) e diferentes quantidades de sal de ferro (Tabela 1). A proporção de ácido sulfúrico utilizada foi baseada em estudos preliminares, considerando que o carvão formado fosse isento de enxofre em sua composição. ${ }^{9}$ Como fonte de ferro foram utilizados o sulfato de ferro (II) heptahidratado $\left(\mathrm{FeSO}_{4} \cdot 7 \mathrm{H}_{2} \mathrm{O}\right.$-Sigma Aldrich) e nitrato de ferro (III) nonahidratado $\left(\mathrm{Fe}\left(\mathrm{NO}_{3}\right)_{3} \cdot 9 \mathrm{H}_{2} \mathrm{O}-\mathrm{Sigma}\right.$ Aldrich). A porcentagem de ferro em cada material foi de 5 e $10 \%$, porcentagem de ferro em relação à massa de carvão obtido. Para o cálculo da massa de carvão obtido, foi considerado um rendimento de $25 \%$ em massa. ${ }^{9}$

Posteriormente, o tratamento térmico foi realizado em um forno horizontal (Sanchis) sob atmosfera de nitrogênio $\left(50 \mathrm{~mL} \mathrm{~min}^{-1}\right)$, utilizando-se uma rampa de aquecimento de $20{ }^{\circ} \mathrm{C} \mathrm{min}^{-1}$, e os materiais foram mantidos em diferentes temperaturas de $500{ }^{\circ} \mathrm{C}$ e $600{ }^{\circ} \mathrm{C}$ por $2 \mathrm{~h}$ ou $4 \mathrm{~h}$.

Após o tratamento térmico, eles foram lavados com água destilada em um extrator Soxhlet para remoção do ferro livre e em seguida foram secos em estufa (Sterilifer SX1.4DTMS) a $100{ }^{\circ} \mathrm{C}$ por $12 \mathrm{~h}$. Os materiais foram macerados e peneirados para homogeneização do tamanho de partícula entre 250 a $500 \mu \mathrm{m}$. A nomeação dos materiais foi realizada de acordo com as variáveis, sendo utilizado o padrão ABCD: $\mathrm{A}=$ sal de ferro utilizado, $\mathrm{N}$ para nitrato de ferro (III) e $\mathrm{S}$ para sulfato de ferro (II); B =\% de ferro, 5 para $5 \%$ ou 10 para $10 \% ; \mathrm{C}=$ tempo, 2 para $2 \mathrm{~h}$ ou 4 para $4 \mathrm{~h} \mathrm{e} \mathrm{D}=$ a temperatura de tratamento térmico, 5 para $500{ }^{\circ} \mathrm{C}$ ou 6 para $600{ }^{\circ} \mathrm{C}$ (Tabela 1 ).

Tabela 1. Nomenclatura dos materiais preparados e quantidade de sal de ferro utilizada

\begin{tabular}{|c|c|c|c|c|c|}
\hline \multicolumn{3}{|c|}{$\mathrm{FeSO}_{4} \cdot 7 \mathrm{H}_{2} \mathrm{O}$} & \multicolumn{3}{|c|}{$\mathrm{Fe}\left(\mathrm{NO}_{3}\right)_{3} \cdot 9 \mathrm{H}_{2} \mathrm{O}$} \\
\hline Composito & $\%$ & Massa (g) & Composito & $\%$ & Massa (g) \\
\hline S525 & 5 & 1,24 & N525 & 5 & 1,81 \\
\hline S526 & 5 & 1,24 & N526 & 5 & 1,81 \\
\hline S545 & 5 & 1,24 & N545 & 5 & 1,81 \\
\hline S546 & 5 & 1,24 & N546 & 5 & 1,81 \\
\hline S1025 & 10 & 2,48 & N1025 & 10 & 3,62 \\
\hline S1026 & 10 & 2,48 & N1026 & 10 & 3,62 \\
\hline S1045 & 10 & 2,48 & N1045 & 10 & 3,62 \\
\hline S1046 & 10 & 2,48 & N1046 & 10 & 3,62 \\
\hline
\end{tabular}

Massa de glicerina $=22,50 \mathrm{~g}$ e massa de ácido sulfúrico $=2,20 \mathrm{~g}$.

\section{Caracterização dos materiais}

Para deteminar a temperatura de tratamento térmico do material, foi realizada uma análise termogravimétrica em atmosfera inerte. As análises foram realizadas em um equipamento Netzsch, modelo TG 209 F1 Phoenix, sob fluxo de $100 \mathrm{~mL} \mathrm{~min}^{-1}$ de nitrogênio com taxa de aquecimento de $20^{\circ} \mathrm{C} \mathrm{min}{ }^{-1}$ até $800{ }^{\circ} \mathrm{C}$. Para a caracterização da quantidade de componentes inorgânicos, teor de cinzas, presente nos diferentes materiais, foi realizada a análise termogravimétrica sob fluxo de $100 \mathrm{~mL} \mathrm{~min}^{-1}$ de ar sintético (atmosfera oxidante), com taxa de aquecimento de $20{ }^{\circ} \mathrm{C} \mathrm{min}^{-1}$ e temperatura final de $800{ }^{\circ} \mathrm{C}$.

O material carbonáceo foi estudado por espectroscopia Raman em um equipamento Horiba, modelo Olympus com uma fonte de laser a $514 \mathrm{~nm}$ e intervalo espectral de 350 a $3800 \mathrm{~cm}^{-1}$.

As características porosas dos materiais foram analisadas por fissisorção de nitrogênio a $-196^{\circ} \mathrm{C}$ em um equipamento Quantchrome Instrument-NOVA 4200. As amostras foram tratadas previamente a $200{ }^{\circ} \mathrm{C}$ por $240 \mathrm{~min}$. A área superficial específica foi calculada usando a equação de Brunauer-Emmett-Teller (BET). ${ }^{27}$

A morfologia dos materiais foi analisada por microscopia eletrônica de varredura acoplando com energia dispersiva de Raios X. As análises foram realizadas utilizando-se um equipamento da marca FEI, Modelo Inspect S50, acoplado com sistema para microanálise química por Espectroscopia por Energia Dispersiva de Raios X (Bruker), operando em $10 \mathrm{kV}$ e $10 \mathrm{~mA}$.

A estrutura cristalina dos materiaiss foi determinada por Difração de Raios X. As análises foram realizadas em um difratômetro Rigaku, modelo Ultima IV, com lâmpada de cobre $(\mathrm{Cu})$ e fonte de energia $40 \mathrm{kV}$, com varredura de 5 a $70^{\circ}$ e velocidade de $5^{\circ} \mathrm{min}^{-1}$.

\section{Remoção de contaminantes orgânicos}

A eficiência dos materiais preparados foi avaliada na remoção de contaminantes orgânicos utilizando-se o composto modelo azul de metileno (AM). Para o teste de remoção pelo processo de oxidação, foram adicionados $10 \mathrm{mg}$ do material em $9,9 \mathrm{~mL}$ de solução de AM, $10 \mathrm{mg} \mathrm{L}^{-1}$ e $0,1 \mathrm{~mL}$ de peróxido de hidrogênio. Para o teste de remoção pelo processo de adsorção foram utilizados $10 \mathrm{mg}$ dos compósitos em $10 \mathrm{~mL}$ da solução $\mathrm{AM}$ de $10 \mathrm{mg} \mathrm{L}^{-1}$. Ambas as soluções foram mantidas sob agitação constante por determinado 
tempo e em seguida centrifugadas por $20 \mathrm{~min}$ a $2500 \mathrm{rpm}$ para separação do sobrenadante. Para determinação da concentração de AM restante em solução, o sobrenadante foi avaliado em um espectrômetro de UV-visível, Jasco V-730, em comprimento de onda de $665 \mathrm{~nm}$. Para cálculo da concentração de AM restante foi utilizada uma curva de calibração preparada com diferentes concentrações, 0,5 a $6 \mathrm{mg} \mathrm{L}^{-1}$. Também foram realizadas medidas de fluorescência no estado estacionário do AM em um espectrofluorímetro da marca Edinburgh Instruments, modelo FS-5, com comprimento de onda de excitação de $615 \mathrm{~nm}$.

\section{RESULTADOS E DISCUSSÃO}

Com intuito de analisar a quantidade de ferro impregnado ao polímero obtido na primeira etapa de preparação, foi realizada a análise termogravimétrica em atmosfera oxidante de ar sintético. A análise da estabilidade térmica para o polímero impregnado foi investigada em atmosfera de nitrogênio. As análises foram conduzidas para o material com $5 \%$ de ferro, utilizando o $\mathrm{FeSO}_{4} \cdot 7 \mathrm{H}_{2} \mathrm{O}$ (Figura 1).

A combustão do material carbonáceo ocorre a partir de $350^{\circ} \mathrm{C} \mathrm{com}$ perda acentuada de massa a partir de $450{ }^{\circ} \mathrm{C}$ (Figura 1a). A quantidade de material inorgânico restante, cinzas, foi de aproximadamente 7,0\% em massa para o ferro/polímero. Para o polímero sem presença de ferro, a quantidade de cinzas foi desprezível. A polimerização das moléculas de glicerol catalisada por ácido é descrita na literatura como um processo de policondensação, pois existem três grupos hidroxilas onde pode ocorrer a polimerização com formação de um polímero ramificado. Medeiros e colaboradores relatam um processo de policondensação por três etapas: iniciação, propagação e terminação, no qual ocorre um processo complexo de formação de um sol-gel até a formação de um polímero rígido. ${ }^{28}$ Neste trabalho verificou-se a formação de polímero rígido, sem diferença aparente em presença de sal de ferro. As cinzas geradas em um tratamento térmico de material carbonáceo em atmosfera oxidante com presença de ferro são compostas basicamente por hematita $\mathrm{Fe}_{2} \mathrm{O}_{3} \cdot{ }^{29,30}$ Portanto, podemos determinar que a quantidade de ferro obtida para o material analisado foi de 4,9\% em massa, semelhante à quantidade teórica utilizada para impregnação desse material, 5,0\% em massa. Essa pequena diferença encontrada entre o valor teórico e experimental pode ser atribuída às perdas ocorridas por adesão do ferro às paredes do reator utilizado para impregnação e tratamento térmico e/ou mesmo devido à precisão da análise termogravimétrica.

Pelos resultados apresentados na Figura 1b, observam-se três eventos bem definidos durante o tratamento térmico em atmosfera de nitrogênio. A decomposição ocorrida em aproximadamente $200{ }^{\circ} \mathrm{C}$ pode ser atribuída à perda de água de coordenação, um evento em $400{ }^{\circ} \mathrm{C}$ pode ser atribuído à decomposição térmica do polímero e posteriomente, no último evento, ocorre a formação da massa de carbono fixa. Levando isso em conta, com intuito de formação de um material estável, foram definidas as temperaturas de $500{ }^{\circ} \mathrm{C}$ e $600{ }^{\circ} \mathrm{C}$ para o tratamento térmico dos materiais ferro/polímero.

Após a preparação de todos os materiais, foi realizada a análise temogravimétrica em atmosfera oxidante para determinação do teor de cinzas. Todos os materiais apresentaram perfil de decomposição semelhante, variando apenas a quantidade de cinzas ou como relatado anteriormente, a quantidade de óxido de ferro. A porcentagem de óxido de ferro para os materiais variou entre 5 a $17 \%$ em massa. Essa procentagem não apresentou uma correlação clara com a temperatura ou tempo de tratamento, apenas mostrou uma pequena coerência com a quantidade nominal de ferro impregnada. Na Figura 2, é apresentado o perfil de decomposição para o N1025 e S1025.

O perfil para a decomposição térmica das amostras N1025 e S1025 foi semelhante, mostrando três etapas bem definidas na faixa de temperatura analisada. Na primeira etapa, os materiais apresentaram alta estabilidade até $450{ }^{\circ} \mathrm{C}$, sendo esse resultado coerente com as condições de tratamento térmico utilizadas. Até a temperatura de $580{ }^{\circ} \mathrm{C}$, foi observada uma perda de massa significativa, cerca de $85 \%$, a qual pode ser atribuída à combustão do material orgânico presente nos materiais. Na última etapa, em estágio de aquecimento superior a $600{ }^{\circ} \mathrm{C}$, não houve alteração substancial na perda de massa. Como relatado anteriormente, a massa resultante é composta por materiais inorgânicos os quais são atribuídos à fase de ferro hematita, $\mathrm{Fe}_{2} \mathrm{O}_{3}$, visto que o carvão preparado a partir do resíduo é isento de componentes inorgânicos. O teor de cinzas foi de 12 e $14 \%$ para S1025 e N1025, respectivamente. Na literatura, são relatados diversos teores de cinzas, pois esses dependem da quantidade de ferro impregnada, da temperatura de tratamento e da matéria prima utilizada como fonte de carbono. Ianos e colaboradores prepararam um material à base de óxido de ferro/nanocompósito de carbono, com teor de cinzas de $20 \% .{ }^{31}$ Cui \& Atkinson prepararam um compósito de ferro/carvão utilizando como matéria prima o glicerol em presença de ácido sulfúrico e/ou ácido fosfórico e posterior tratamento térmico com $\mathrm{CO}_{2}$. Os valores de cinzas encontrados foram de 10 a $14 \%$, semelhantes aos encontrados neste trabalho. ${ }^{32}$

Com intuito de avaliar o tipo de estrutura carbonácea formada, os materiais foram analisados por espectroscopia Raman. Os resultados para N1025 e S1025 são apresentados na Figura 2b. Pelos resultados, verificamos duas bandas características, G e D em 1100 a $1800 \mathrm{~cm}^{-1}$,
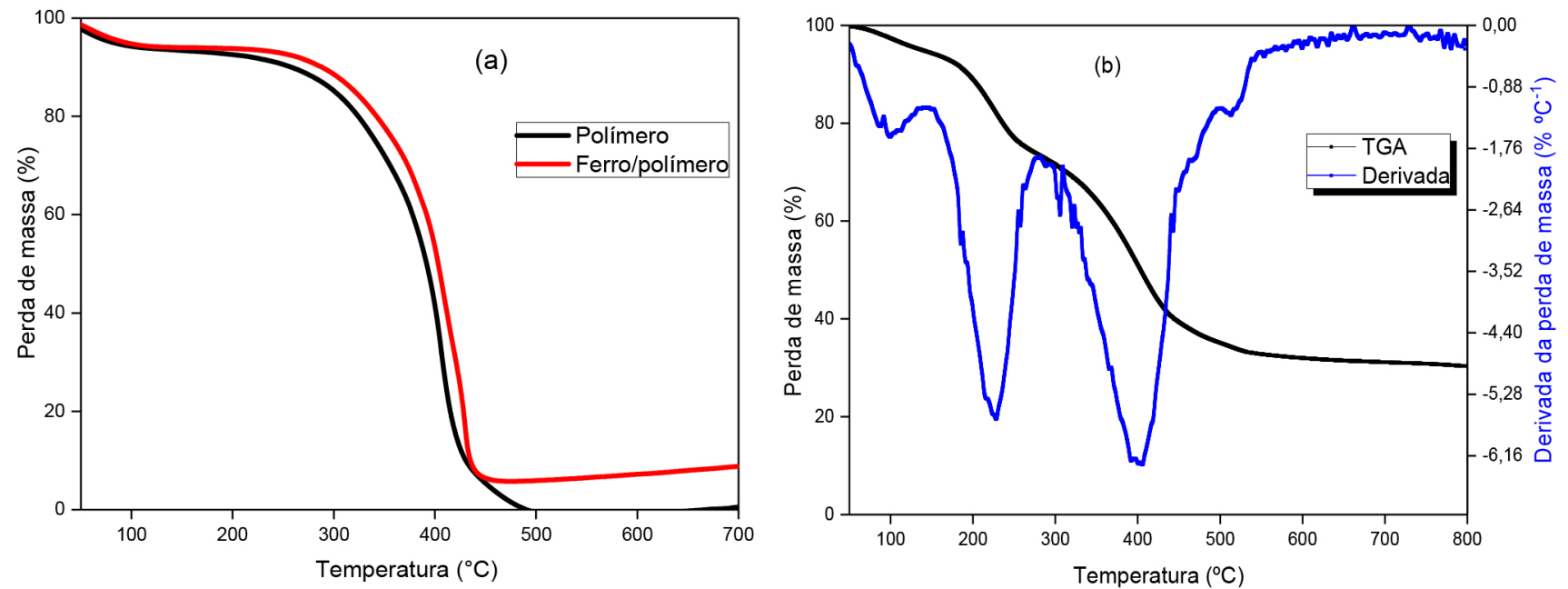

Figura 1. Análise termogravimétrica do polímero impregnado $\left(5 \%\right.$ com $\left.\mathrm{FeSO}_{4} \cdot 7 \mathrm{H}_{2} \mathrm{O}\right)$ : (a) em atmosfera oxidante de ar sintético e (b) em atmosfera de nitrogênio 

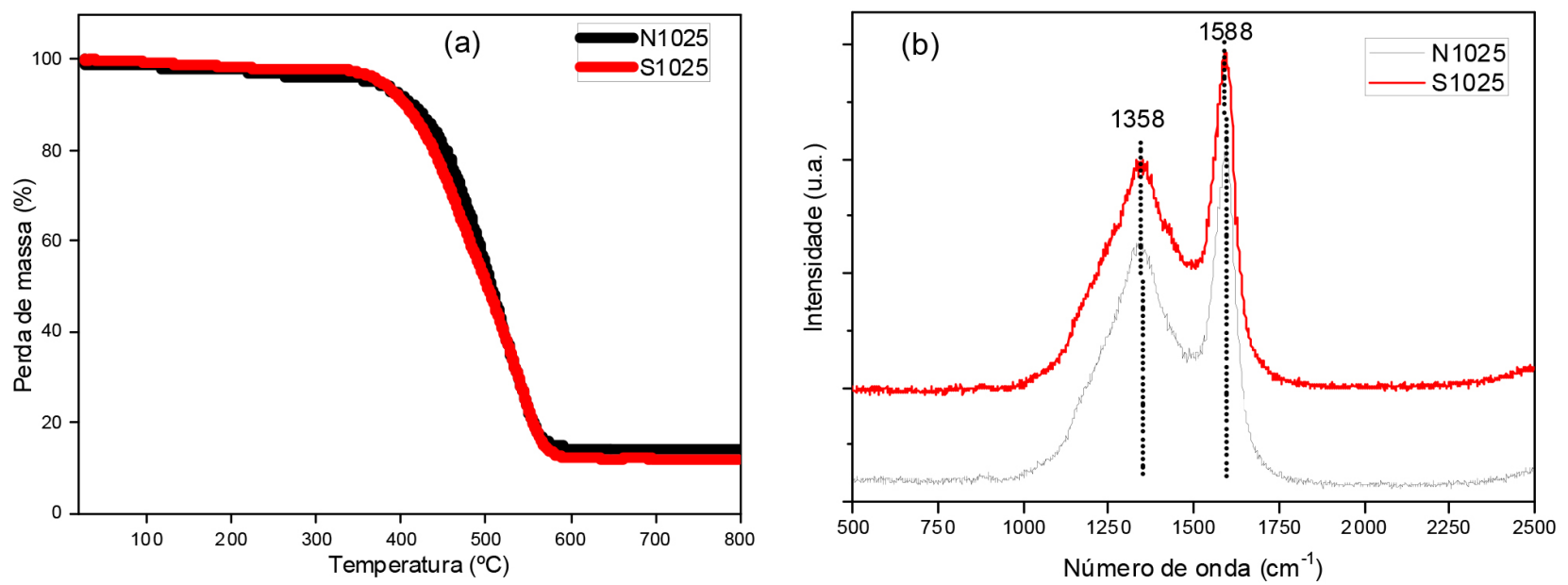

Figura 2. (a) Análise termogravimétrica em atmosfera de ar sintético para os materiais N1025 e S1025 (b) espectros Raman para os materiais N1025 e S1025

típicas de hidrocarbonetos policíclicos de material amorfo. A banda D, em $1358 \mathrm{~cm}^{-1}$, é característica da estrutura grafítica desordenada. A banda em $1588 \mathrm{~cm}^{-1}$ é designada banda $\mathrm{G}$, que corresponde à estrutura grafítica ${ }^{33}$

A integração da área de cada banda permite o cálculo da razão da intensidade $\mathrm{I}_{\mathrm{D}} / \mathrm{I}_{\mathrm{G}}$, e quanto maior o valor mais desorganizado é o material. Os valores encontrados neste trabalho mostraram que o valor da razão $I_{D} / I_{G}$ variou de 0,72 a 0,77 para todos os materiais preparados. Verificou-se que ocorreu uma diminuição do valor da razão $\mathrm{I}_{\mathrm{D}} / \mathrm{I}_{\mathrm{G}} \mathrm{com}$ o aumento do tempo ou temperatura de tratamento térmico. Isso pode ser devido ao maior ordenamento da estrutura carbonácea que ocorre com o fornecimento de energia, seja pelo aumento da temperatura ou pelo tempo de tratamento. Os valores para o N1025 e S1025, materiais preparados com as mesmas condições de tratamento térmico, foram semelhantes, obtendo 0,77 e 0,76, respectivamente. Esse resultado mostra que a fonte de ferro não interferiu no grau de ordenamento do material. Resultados superiores foram encontrados por Baek e colaboradores que encontraram valores de $\mathrm{I}_{\mathrm{D}} / \mathrm{I}_{\mathrm{G}}$ de 0,92 a 0,98 . Os autores observaram que com maior temperatura de tratamento dos materiais carbonáceos obtem maior grau de desordem e atribuíram este fato à inserção de heterátomos na estrutura do carvão. ${ }^{34}$

A área superficial específica obtida para todos os materiais foi inferior a $30 \mathrm{~m}^{2} \mathrm{~g}^{-1}$. Esse resultado mostra que o tratamento térmico não foi eficaz na formação de porosidade no material carbonáceo a partir de glicerina bruta em presença de sal de ferro. Na literatura, é relatada a obtenção de materiais com elevada área superficial utilizando sais de ferro pela impregnação de diferentes precursores e posterior tratamento térmico. Xu e colaboradores descreveram a ativação de resíduos de poliéster na presença de $\mathrm{FeCl}_{2}, \mathrm{FeCl}_{3}$ e $\mathrm{FeSO}_{4}$. $\mathrm{A}$ área superficial obtida para o material preparado em presença de cloretos foi aproximadamente $1400 \mathrm{~m}^{2} \mathrm{~g}^{-1}$, enquanto a área superficial para o material ativado em presença de sulfato foi menor, $382 \mathrm{~m}^{2} \mathrm{~g}^{-1}$. Essa diferença foi atribuída à importância do cloro na formação da porosidade.$^{29} \mathrm{Li}$ e colaboradores relatam a obtenção de materiais à base de ferro/carvão utilizando como precursor a celulose, com área superficial especifica menor que $100 \mathrm{~m}^{2} \mathrm{~g}^{-1} \cdot{ }^{35}$ Cui e Atkinson relatam a preparação de materiais carbonáceos a partir de glicerina e ativação combinada com cloreto férrico/ácido sulfúrico/ $\mathrm{CO}_{2}$ e/ou cloreto férrico/ácido fosfórico/ $/ \mathrm{CO}_{2}$, obtendo área superficial específica de 426 e $561 \mathrm{~m}^{2} \mathrm{~g}^{-1}$, respectivamente. No entanto, foi utilizado um método de preparação em duas etapas: tratamento térmico a $600{ }^{\circ} \mathrm{C}$ em presença de atmosfera inerte de nitrogênio e posteriormente em $900{ }^{\circ} \mathrm{C}$ em presença de $\mathrm{CO}_{2} \cdot{ }^{32}$ Os autores também relatam a preparação de materiais com tratamento térmico com ácido sulfúrico/ $/ \mathrm{CO}_{2}$ e/ou ácido fosfórico/ $\mathrm{CO}_{2}$ obtendo área superficial específica de 509 e $406 \mathrm{~m}^{2} \mathrm{~g}^{-1}$, respectivamente. Esses resultados evidenciam que a presença de ferro não influenciou significativamente na área superficial especifica obtida. ${ }^{32}$ Assim, é evidente que a elevada temperatura e a atmosfera de $\mathrm{CO}_{2}$ foram essenciais para formação de estruturas porosas em materiais utilizando matéria prima que contenha alto teor de glicerol. ${ }^{32}$ Os materiais preparados neste trabalho também evidenciam que as condições utilizadas para o tratamento térmico, utilizando atmosfera inerte $\left(\mathrm{N}_{2}\right)$ e sal de ferro, não são eficientes para o desenvolvimento de porosidade nos materiais obtidos ou o óxido de ferro formado está bloqueando os poros.

Com intuito de verificar o bloqueio dos poros, o compósito N1025 foi lavado com solução de ácido clorídrico $5 \mathrm{~mol} \mathrm{~L}^{-1}$ para remoção do ferro e posteriormente com água destilada. A área superficial especifica obtida foi de $170 \mathrm{~m}^{2} \mathrm{~g}^{-1}$ evidenciando o bloqueio de parte da porosidade pela grande quantidade de óxido de ferro presente, 14\%. Porém, a área superficial específica ainda é considerada baixa para materiais carbonáceos quando comparamos com outros materiais relatados na literatura, os quais apresentam área superficial específica acima de $400 \mathrm{~m}^{2} \mathrm{~g}^{-1}{ }^{29,32}$

Mesmo sem a obtenção de materiais com alta área superficial específica, eles apresentam grande quantidade de óxido de ferro, podendo ser promissores catalisadores em reações de oxidação de contaminantes orgânicos. O uso de óxido de ferro como catalisador heterogêneo é relatado na literatura em reações de oxidação "tipo Fenton" para degradação de diversos contaminantes orgânicos..$^{36-38}$ Levando isso em consideração, a eficiência dos materiais preparados foi avaliada na remoção de contaminantes orgânicos utilizando o azul de metileno (MB) como composto modelo, via processo de adsorção e oxidação em presença de peróxido de hidrogênio, tipo Fenton. Os resultados obtidos para todos os materiais são apresentados na Figura 3.

A remoção do AM pelo processo de adsorção foi baixa na presença da maioria dos materiais, de 4 a $20 \%$. No entanto, os materiais $\mathbf{S 1 0 2 5}$ e $\mathbf{N 1 0 2 5}$ apresentaram um comportamento diferente, com aproximadamente $40 \%$ de adsorção do AM presente em solução.

O processo de oxidação mostrou maior eficiência, removendo de 20 a $80 \%$ do AM em presença da maioria dos materiais, atingindo 99\% em presença de S1025 e N1025. Não há uma tendência clara para a oxidação mais elevada em presença desses materiais. No entanto, podemos atribuir ao um efeito somático da quantidade de ferro e da área superficial específica. A quantidade de óxido de ferro, cinzas, para S1025 e N1025 foi de 12 e 14\% e a área superficial específica foi

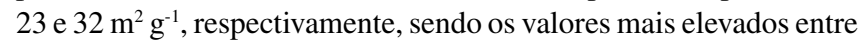
os materiais preparados. Existe um entendimento geral de que há três fatores que influenciam a eficiência de um processo de oxidação via 

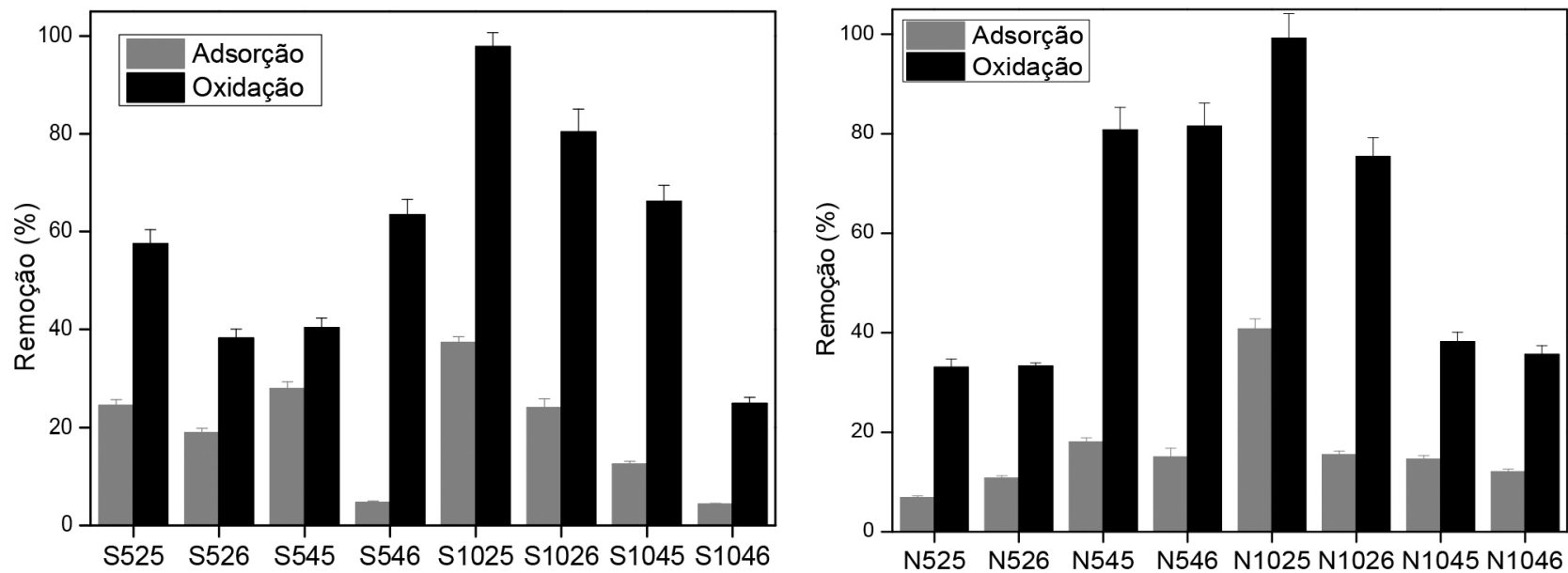

Figura 3. Avaliação da capacidade de remoção de AM para todos os materiais preparados. Condições: Tempo de contato $=24 \mathrm{~h}$, temperatura $25{ }^{\circ} \mathrm{C}$, $10 \mathrm{mg}$ de catalisador e $[A M]=10 \mathrm{mg} \mathrm{L}^{-1}$

Fenton heterogêneo: a fase de ferro presente, a quantidade de ferro superficial e a área superficial específica dos materiais. A oxidação de um composto orgânico pela reação Fenton ocorre via radicais hidroxila $\left({ }^{\circ} \mathrm{OH}\right)$ ou hidroperóxido $\left({ }^{\bullet} \mathrm{OOH}\right)$ que se formam de acordo com a fase de ferro presente no catalisador. ${ }^{19} \mathrm{~A}$ maior área superficial propicia a transferência de massa da solução de AM, ocorrendo maior adsorção do contaminante na superficie do catalisador e consequentemente intensificação do processo de oxidação. ${ }^{39}$

Para determinação da quantidade de ferro superficial, ou seja, a fase ativa dos materiais, a superfície dos materiais S1025 e N1025 foi analisada por microscopia eletrônica de varredura acoplada a energia dispersiva de raios X (MEV-EDS) (Figura 4). O teor de ferro na superfície do material foi de $25 \%$ e 45 e para o S1025 e N1025, respectivamente. Verifica-se que o ferro se encontra disperso na superfície do material carbonáceo, porém parte deste está bloqueando os poros, corroborando com os resultados encontrados para o teste de lavagem com ácido para eliminação do ferro e liberação da porosidade.

As estruturas cristalinas dos materiais S1025 e N1025 foram analisadas por difratometria de raios $\mathrm{X}$ (DRX) com intuito de verificar a fase de ferro presente no material. Os resultados obtidos são apresentados na Figura 5.
Os difratogramas dos materiais são característicos de materiais pouco cristalino ou com cristalitos de tamanho reduzido. Observamse picos em aproximadamente em $2 \theta=24,2^{\circ} ; 33,2^{\circ} ; 35,7^{\circ}$ e $54,1^{\circ}$ para ambos os compóstos o qual pode ser atribuído à formação de hematita $\left(\mathrm{Fe}_{2} \mathrm{O}_{3}\right)$ e picos em $2 \theta=30,2^{\circ} ; 35,7^{\circ} ; 43,2^{\circ} ; 57,2^{\circ}$ e $62,9^{\circ}$, característico de magnetita $\left(\mathrm{Fe}_{3} \mathrm{O}_{4}\right)$.

A formação da hematita pode ser explicada pelo tratamento térmico, no qual podemos observar a transformação do sulfato em temperaturas superiores a $450{ }^{\circ} \mathrm{C}$. Após a primeira reação de desidratação ocorre a formação da hematita de acordo com a equação: $6 \mathrm{FeSO}_{4}(\mathrm{~s}) \rightarrow \mathrm{Fe}_{2}\left(\mathrm{SO}_{4}\right)_{3}+2 \mathrm{Fe}_{2} \mathrm{O}_{3}(\mathrm{~s})+2 \mathrm{SO}_{2}{ }^{40}$

A formação da magnetita é devido à alta temperatura e tempo de tratamento térmico, no qual ocorre a redução parcial do ferro em presença de materiais à base de carbono. Esses resultados estão de acordo com dados relatados da literatura, nos quais é relatado que em temperaturas superiores a $650{ }^{\circ} \mathrm{C}$ ocorre a formação de magnetita, produzida pela redução da hematita em presença de carvão de acordo com a reação: $6 \mathrm{Fe}_{2} \mathrm{O}_{3}(\mathrm{~s})+\mathrm{C}(\mathrm{s}) \rightarrow 4 \mathrm{Fe}_{3} \mathrm{O}_{4}(\mathrm{~s})+\mathrm{CO}_{2}(\mathrm{~g}) .{ }^{41}$

Devido à presença de ferro reduzido na fase magnetita, esses materiais apresentaram melhor atividade catalítica na degradação do AM ocorrendo a formação de radicais hidroxilas.

Outro fator importante em processos de remoção de contaminantes
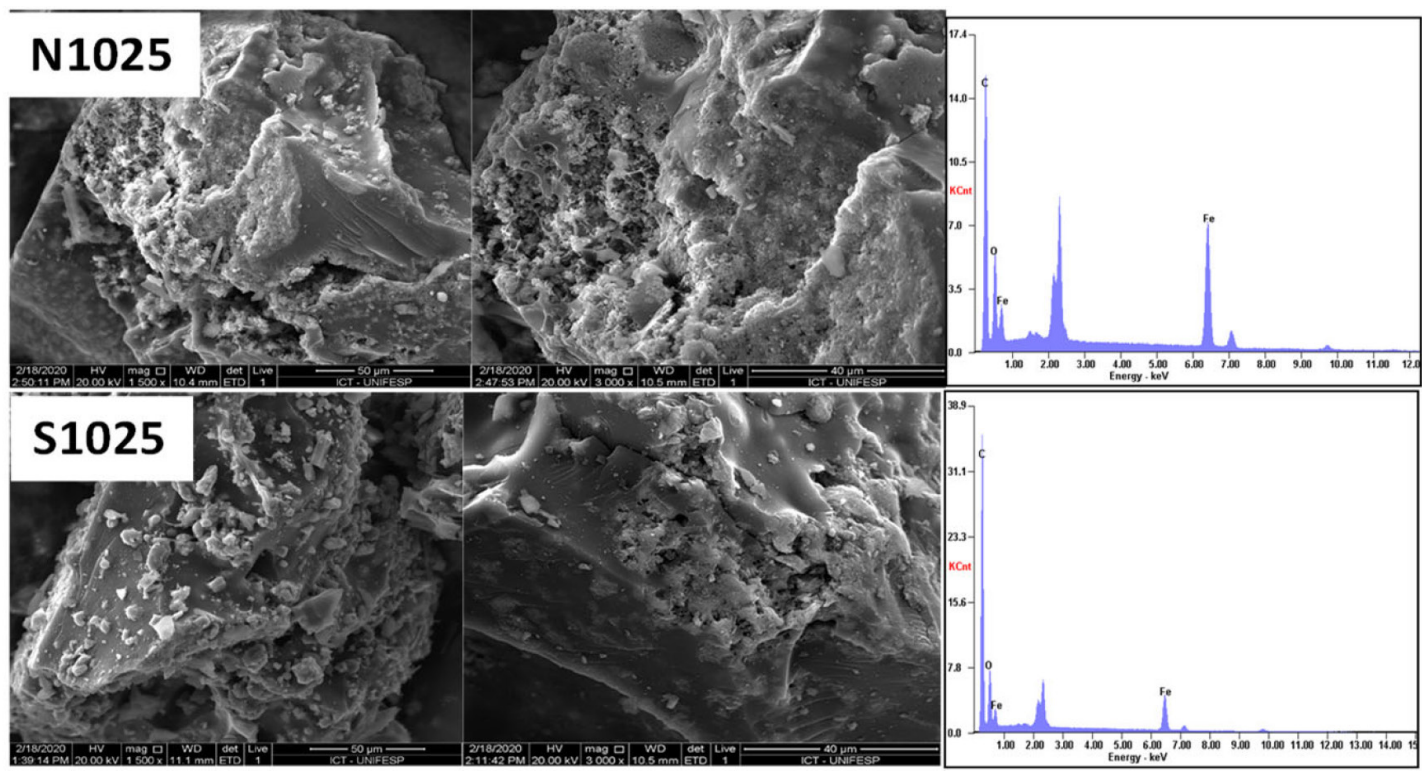

Figura 4. Microscopia eletrônica de varredura com energia dispersiva de raios X para os materiais N1025 e S1025 


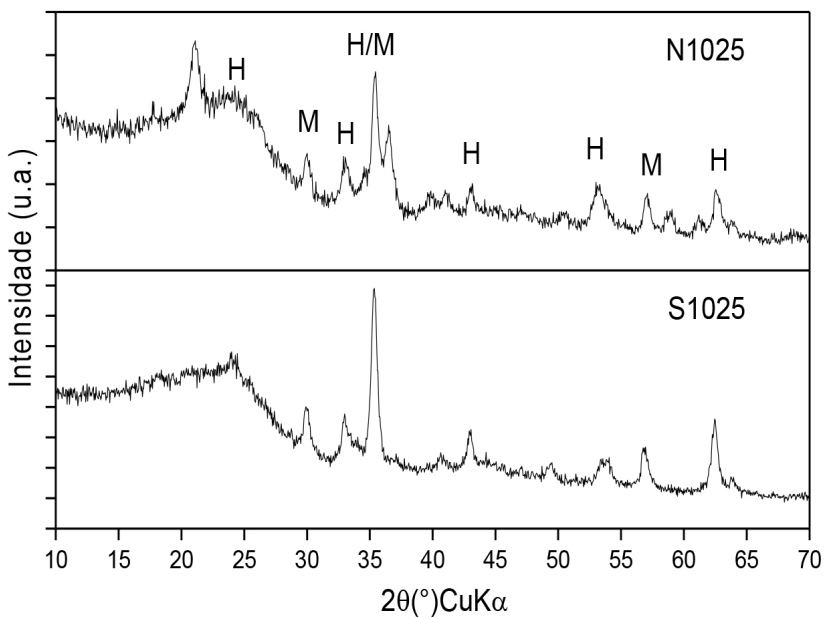

Figura 5. Difratogramas de raios X para as amostras N1025 e S1025. M=Magnetita e H=Hematita

é a velocidade com que esses são removidos, ou seja, a cinética de reação seja por oxidação ou adsorção. Os resultados obtidos para a cinética de remoção do AM em presença de $\$ 1025$ e N1025 e são apresentados nas Figuras 6a e 6b.

A cinética de adsorção de AM para ambos os materiais foi semelhante, atingindo o equilíbrio dinâmico após $4 \mathrm{~h}$. A rápida adsorção pode ser explicada pois os materiais apresentam baixa porosidade e, portanto, toda a superfície satura rapidamente. Resultados semelhantes foram relatados por Honorato e colaboradores em teste de adsorção do AM em materiais de baixa porosidade, alcançando o equilíbrio em $4 \mathrm{~h}$ de contato. ${ }^{42}$

Por outro lado, a cinética de oxidação do AM mostrou resultados diferentes. O $\$ 1025$ mostrou uma baixa atividade, até $8 \mathrm{~h}$ removendo aproximadamente $35 \%$ do AM presente na solução. No entanto, no período entre 8 e $10 \mathrm{~h}$ de reação ocorre uma rápida oxidação, atingindo $90 \%$. A oxidação do AM em presença do N1025 foi rápida com remoção de aproximadamente $75 \%$ em 6 h de reação. Após $10 \mathrm{~h}$ de reação, ambos os catalisadores apresentam $99 \%$ de remoção de AM sem diferença significativa entre ambos os catalisadores. A velocidade inicial para as reações foi de $0,039 \mathrm{~h}^{-1}$ e $0,009 \mathrm{~h}^{-1}$ para o $\mathrm{N1025}$ e S1025, respectivamente. Essa diferença pode ser atribuída à presença de maior quantidade de ferro reduzido na superfície do N1025, ocorrendo a formação de radicais hidroxilas nas primeiras horas de reação. Resultados semelhantes para oxidação de AM

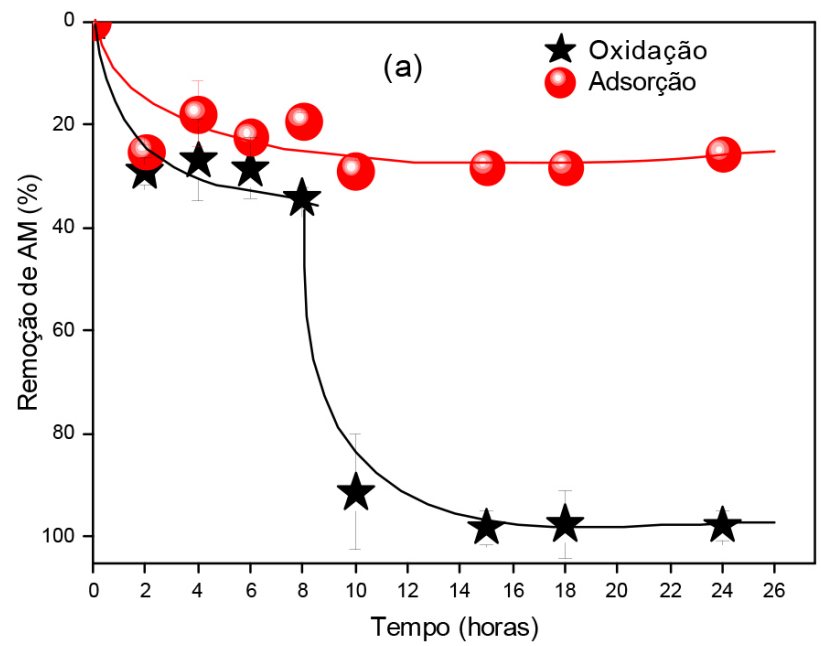

foram relatados por Augusto e colaboradores quando utilizaram um catalisador à base de ferro, obtendo uma baixa remoção inicial. Porém, o máximo de remoção relatado pelos autores foi de $75 \%$ após $20 \mathrm{~h}$, o que foi atribuído pela fase de ferro presente nos catalisadores..$^{43} \mathrm{E}$ consenso entre diversos estudos que catalisadores com pesença de $\mathrm{Fe}_{3} \mathrm{O}_{4}$ apresentam maior atividade que o $\mathrm{Fe}_{2} \mathrm{O}_{3}$ pois geram radicais hidroxila, o qual possui maior potencial oxidante. ${ }^{41,43-45}$ A presença de ferro reduzido $\left(\mathrm{Fe}^{+2}\right)$ nessa fase de ferro faz com que ocorra uma rápida formação de radicais hidroxila e posterior degradação do corante em detrimento da menor velocidade de formação de radicais hidroperóxido (equação 1 e 2). ${ }^{46}$

$$
\begin{array}{ll}
\mathrm{H}_{2} \mathrm{O}_{2}+\mathrm{Fe}^{2+} \rightarrow \mathrm{Fe}^{3+}+\cdot \mathrm{OH}+\cdot \mathrm{OH} & \mathrm{k} \sim 70 \mathrm{M}^{-1} \mathrm{~s}^{-1} \\
\mathrm{H}_{2} \mathrm{O}_{2}+\mathrm{Fe}^{3+} \rightarrow \mathrm{Fe}^{2+}+\cdot{ }^{\circ} \mathrm{OOH}+\mathrm{H}^{+} & \mathrm{k}=0,002-0,01 \mathrm{M}^{-1} \mathrm{~s}^{-1}
\end{array}
$$

Também foi analisada a cinética de oxidação para o AM em presença do catalisador N1025 via espectroscopia de absorção na região do visível de 400 a $800 \mathrm{~nm}$. O espectro de absorbância, Figura 7, apresenta duas bandas características: uma em $615 \mathrm{~nm}$, referente a forma dimérica (D) e outra em $663 \mathrm{~nm}$, atribuída aos monômeros (M) e é corresponde ao grupo cromóforo do corante (ligação dimetilamino). Os anéis benzênicos da molécula apresentam bandas características por volta de 245 e $290 \mathrm{~nm}$, mas que não são aqui apresentadas devido ser também a região de absorção de peróxido de hidrogênio. Em solução aquosa, o corante AM encontra-se em equilíbrio entre as formas diméricas e monoméricas. ${ }^{47}$ Após o processo de oxidação do AM a absorbância diminui proporcionalmente ao tempo de reação, evidenciando à degradação do corante. A redução da banda em 665 nm é atribuída a N-desmetilação do AM, processo responsável pela clivagem do grupo cromóforo, eliminando a coloração da solução. ${ }^{47}$

Foram realizadas medidas de emissão de fluorescência com excitação em $615 \mathrm{~nm}$. Os resultados obtidos são apresentados na Figura 8.

O espectro de emissão de fluorescência do AM antes da reação apresenta duas bandas com máximos em aproximadamente $693 \mathrm{~nm}$ e $755 \mathrm{~nm}$. A intensidade de fluorescência do cromóforo em solução diminui com o tempo de reação, evidenciando a degradação do AM. Além disso, através dos espectros apresentados na Figura 8 (b), é possível verificar um deslocamento para o azul da banda em $693 \mathrm{~nm}$ após a reação oxidativa, sendo que após 24 h de reação, o máximo da banda encontra-se $683 \mathrm{~nm}$. Esse deslocamento é acompanhado da diminuição de intensidade relativa da banda em

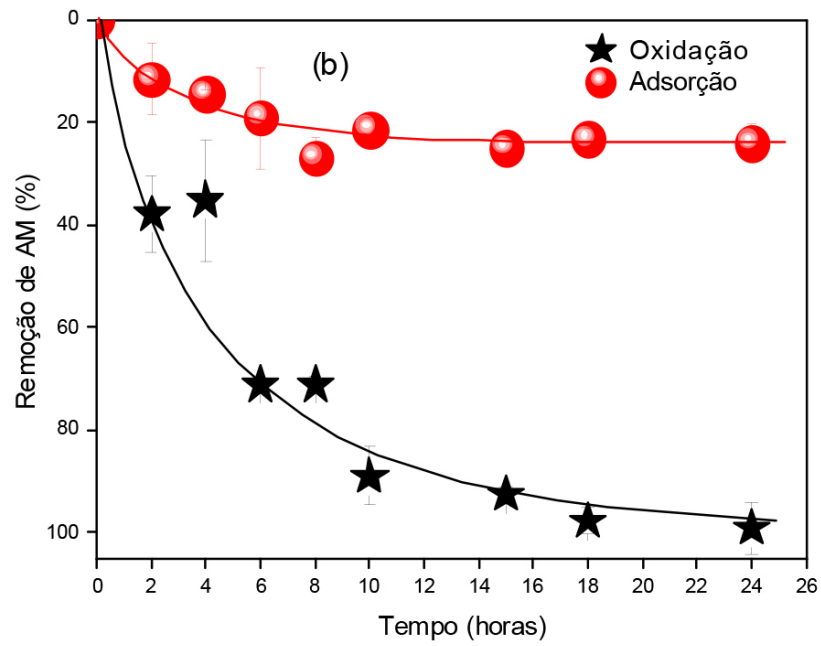

Figura 6. Cinética de remoção para o Azul de Metileno em presença dos materiais (a) S1025 e (b) N1025. Condições: temperatura $25^{\circ} \mathrm{C}$, $10 \mathrm{mg}$ de catalisador $e[A M]=10 \mathrm{mg} \mathrm{L}^{-1}$ 


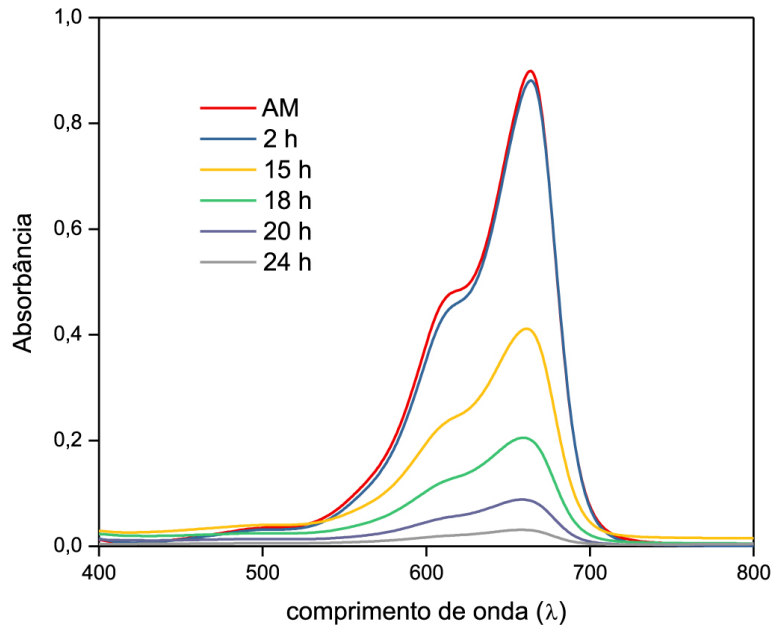

Figura 7. Espectros de absorção do AM em presença do N1025 após diferentes tempos de reação. $[A M]=5 \mathrm{mg} \mathrm{L}^{-1}$

$750 \mathrm{~nm}$, evidenciando um processo de desagregação ocorrida pela diminuição da concentração do corante..$^{47}$

Além disso, a estabilidade do catalisador e seu reaproveitamento são fatores muito importantes em catálise heterogenea. Assim, o

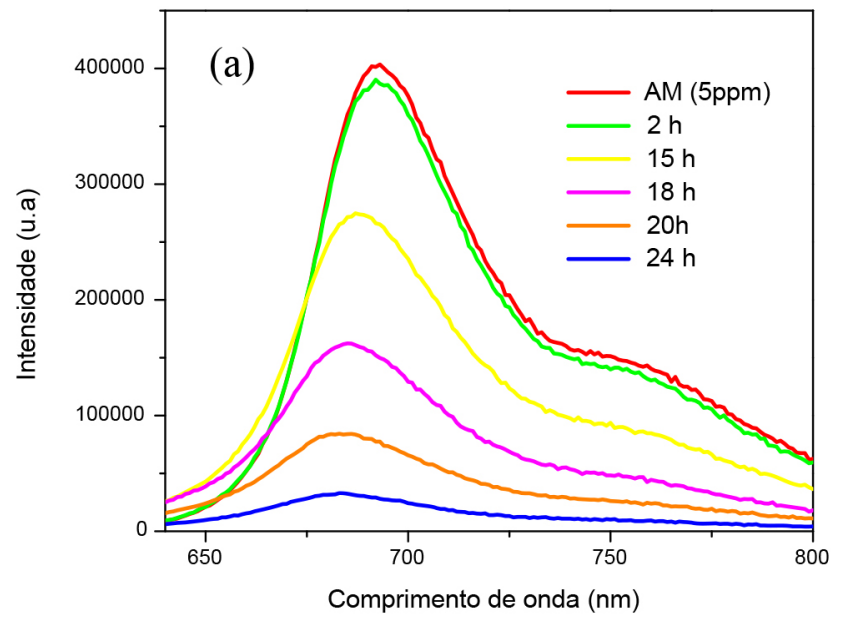

catalisador com maior atividade inicial, N1025, foi avaliado quanto a sua estabilidade utilizando o teste de lixiviação, o qual consiste na remoção do catalisador após determinado tempo e continuidade da avaliação do procesos de oxidação do corante. ${ }^{48}$ Caso ocorra a lixiviação da fase ativa para a solução, seja como $\mathrm{Fe}^{+2}$ ou $\mathrm{Fe}^{+3}$, o processo de oxidação continuará via Fenton homogêneo, pois existe peróxido de hidrogênio em solução. Por outro lado, se o compósito possuir estabilidade e não ocorrer lixiviação, da fase ativa para a solução, o processo de oxidação será interrompido com a remoção do catalisador. ${ }^{48}$ Pelos resultados obtidos, Figura 9a, verifica-se que após a remoção do catalisador, o processo de oxidação foi interrompido, portanto não há fase ativa em solução. $\mathrm{O}$ resultado revelou que o compósito N1025 apresenta alta estabilidade em meio aquoso e o processo de oxidação do AM ocorre via processo heterogêneo.

O reaproveitamento do catalisador, ou seja, a eficiência catalítica do material em vários ciclos consecutivos de oxidação foi realizada para o N1025 e o resultado obtido é apresentado na Figura 9(b). O catalisador apresentou eficiência em até quatro ciclos, não perdendo sua atividade catalítica o que evidencia que este material é promissor para utilização em processos industriais. Vale ressaltar que não foi realizado nenhum tratamento em cada etapa, apenas remoção do sobrenadante e secagem do catalisador para posterior início do processo de oxidação. $\mathrm{O}$ reaproveitamento de catalisadores ácidos

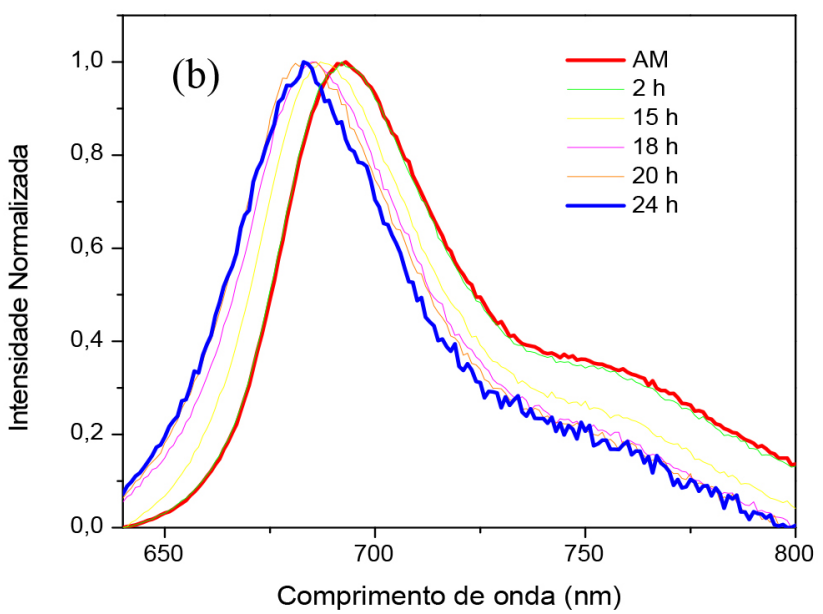

Figura 8. (a) Espectros de emissão de fluorescência da solução padrão de AM em presença do N1025 e (b) Espectros de emissão de fluorescência normalizados. $\lambda_{\text {exc }}=615 \mathrm{~nm}$
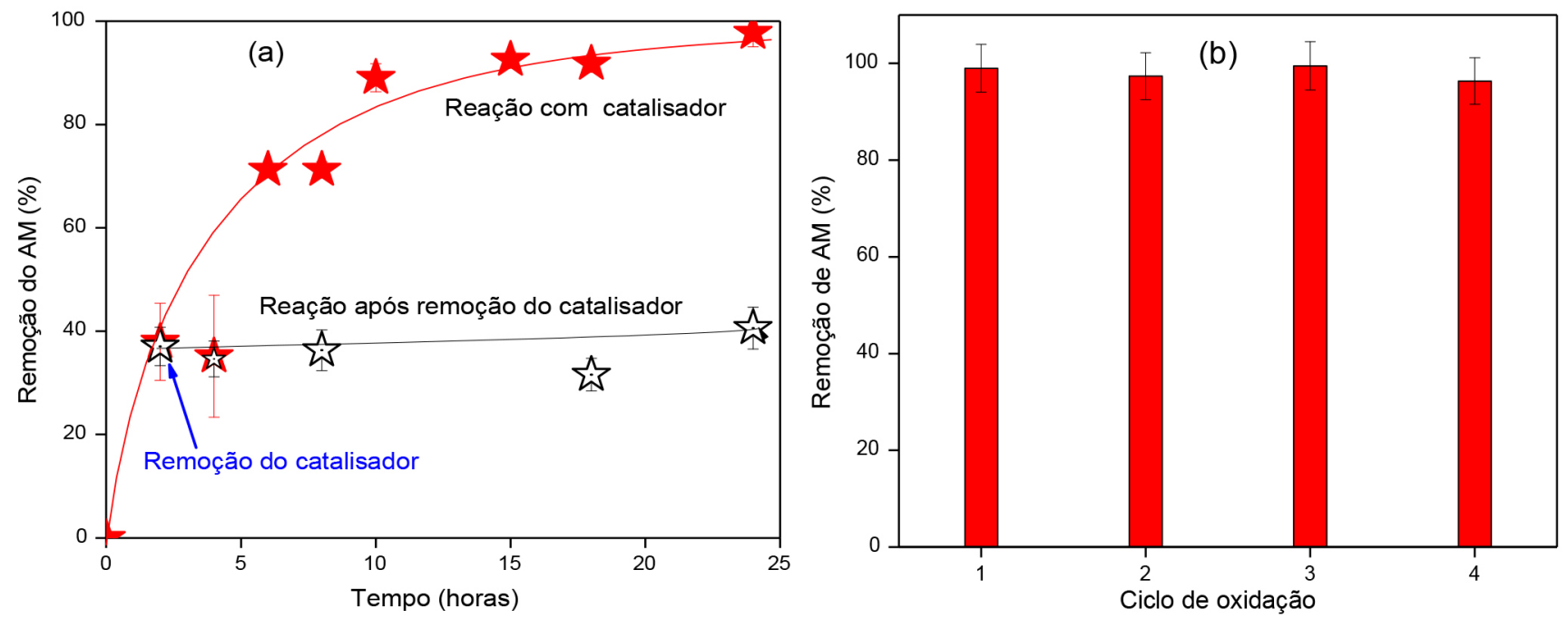

Figura 9. (a) Teste de lixiviação do catalisador $N 1025$ e (b) reaproveitamento do catalisador N1025. Condições: temperatura $25^{\circ} \mathrm{C}, 10 \mathrm{mg}$ de catalisador, [AM] $=10 \mathrm{mg} \mathrm{L}^{-1}$ e tempo de $24 \mathrm{~h}$ 
obtidos a partir de resíduos do biodiesel é um importante fator para aproveitamento deste resíduo, sendo descrito em outros trabalhos mostrados na literatura. ${ }^{8,49}$

\section{CONCLUSÕES}

O aproveitamento da glicerina, resíduo do biodiesel, para produção de materiais à base de ferro/carvão mostrou-se um processo viável. A carbonização utilizando $\mathrm{FeSO}_{4} \cdot 7 \mathrm{H}_{2} \mathrm{O}$ e $\mathrm{Fe}\left(\mathrm{NO}_{3}\right)_{3} \cdot 9 \mathrm{H}_{2} \mathrm{O}$ gerou materiais com baixa área superficial específica, aproximadamente $32 \mathrm{~m}^{2} \mathrm{~g}^{-1}$ e com grande quantidade de óxido de ferro na superfície. Os óxidos de ferro presentes na superfície foram identificados como hematita e magnetita. Os materiais mostraram-se eficientes, sendo que o material N1025 apresentou melhor desempenho com remoção de $99 \%$ AM, via oxidação tipo Fenton. O aproveitamento da glicerina para produção de materiais à base de ferro/carvão pelo processo in situ mostrou-se um processo viável formando um material com estabilidade, não ocorrendo à lixiviação da fase ativa para a solução, comprovando o processo de catálise heterogêneo. Além disso, o catalisador mostrou-se ativo até o quarto ciclo analisado, sendo viável seu reaproveitamento.

\section{AGRADECIMENTOS}

Nossos agradecimentos ao CNPQ pelo apoio financeiro (processo $\mathrm{n}^{\circ}$ 401796/2016-7) e NAPCEM/UNIFESP pela condução das análises.

\section{REFERENCIAS}

1. Leoneti, A. B.; Aragão-Leoneti, V.; Oliveira, S. V. W. B.; Renewable Energy 2012, 45, 138.

2. Karinen, R. S.; Krause, A. O. I.; Appl. Catal., A 2006, 306, 128.

3. Harter, L. V. L.; Santos, D. Q.; Fabris, J. D.; Quim. Nova 2019, 42, 143.

4. http://www.anp.gov.br/?id=470, acessada em fevereiro 2020.

5. Costa, L. G.; Sitoe, B. V.; Santos, D. Q.; Borges Neto, W.; Quim. Nova 2020, 43, 723.

6. Mota, C. J. A.; Silva, C. X. A. d. and Gonçalves, V. L. C.; Quim. Nova 2009, 32, 639.

7. Ribeiro, R. S.; Silva, A. M. T.; Pinho, M. T.; Figueiredo, J. L.; Faria, J. L.; Gomes, H. T.; Catal. Today 2015, 240, 61.

8. Mantovani, M.; Mandelli, D.; Gonçalves, M.; Carvalho, W. A.; Chem. Eng. J. 2018, 348, 860.

9. Gonçalves, M.; Mantovani, M.; Carvalho, W. A.; Rodrigues, R.; Mandelli, D.; Silvestre Albero, J.; Chem. Eng. J. 2014, 256, 468.

10. Prabhavathi Devi, B. L. A.; Gangadhar, K. N.; Siva Kumar, K. L. N.; Shiva Shanker, K.; Prasad, R. B. N.; Sai Prasad, P. S.; J. Mol. Catal. A: Chem. 2011, 345, 96.

11. Prabhavathi Devi, B. L.; Gangadhar, K. N.; Sai Prasad, P. S.; Jagannadh, B.; Prasad, R. B.; ChemSusChem 2009, 2, 617.

12. Medeiros, M. d. A.; Cançado, T. M.; Leite, C. M. M.; Lago, R. M.; J. Chem. Technol. Biotechnol. 2012, 87, 1654.

13. Medeiros, M. A.; Ardisson, J. D.; Lago, R. M.; J. Chem. Technol. Biotechnol. 2020, 95, 1045.

14. Prabhavathi Devi, B. L. A.; Vijai Kumar Reddy, T.; Vijaya Lakshmi, K.; Prasad, R. B. N.; Bioresour. Technol. 2014, 153, 370.

15. Pattanaik, L.; Duraivadivel, P.; Hariprasad, P.; Naik, S. N.; Bioresour. Technol. 2020, 301, 122721.

16. Rey, A.; Faraldos, M.; Casas, J. A.; Zazo, J. A.; Bahamonde, A.; Rodriguez, J. J.; Appl. Catal., B 2009, 86, 69.
17. Santana, C. S.; Velloso, C. C. V.; Aguiar, A.; Quim. Nova 2019, 42, 149.

18. Pedro-Cedillo, L. S.; Méndez-Novelo, R. I.; Hernández-Núñez, E.; Giácoman-Vallejos, G.; Bassam, A.; Quim. Nova 2019, 42, 418.

19. Fenton, H. J. H.; J. Chem. Soc., Trans. 1894, 65, 899.

20. Munoz, M.; de Pedro, Z. M.; Casas, J. A.; Rodriguez, J. J.; Appl. Catal., B 2015, 176, 249 .

21. Bello, M. M.; Abdul Raman, A. A.; Asghar, A.; Process Saf. Environ. Prot.2019, 126, 119 .

22. Yao, Y.; Wang, L.; Sun, L.; Zhu, S.; Huang, Z.; Mao, Y.; Lu, W.; Chen, W.; Chem. Eng. Sci. 2013, 101, 424.

23. Wu, J.; Gao, H.; Yao, S.; Chen, L.; Gao, Y.; Zhang, H.; Sep. Purif. Technol. 2015, 147, 179.

24. Yang, N.; Liu, Y.; Zhu, J.; Wang, Z.; Li, J.; Chemosphere 2020, 252, 126333.

25. Zhu, Y.; Zhu, R.; Xi, Y.; Zhu, J.; Zhu, G.; He, H.; Appl. Catal., B 2019 , 255, 117739.

26. Manfron, S.; Thomé, A.; Cecchim, I.; Reddy, K. R.; Quim. Nova 2020, $43,623$.

27. Brunauer, S.; Emmett, P. H.; Teller, E.; J. Am. Chem. Soc. 1938, 60, 309.

28. Medeiros, M. A.; Lago, R. M.; Quim. Nova 2011, 34, 1079.

29. Xu, Z.; Tian, D.; Sun, Z.; Zhang, D.; Zhou, Y.; Chen, W.; Deng, H.; Colloids Surf., A 2019, 565, 180.

30. Müller, M.; Villalba, J.; Anaissi, F.; Semina: Cienc. Exatas Tecnol. 2014, $35,9$.

31. Ianoş, R.; Păcurariu, C.; Muntean, S. G.; Muntean, E.; Nistor, M. A.; Nižňanský, D.; J. Alloys Compd. 2018, 741, 1235.

32. Cui, Y.; Atkinson, J. D.; Chemosphere 2019, 228, 694.

33. Sadezky, A.; Muckenhuber, H.; Grothe, H.; Niessner, R.; Pöschl, U.; Carbon 2005, 43, 1731.

34. Baek, J.; Shin, H. S.; Chung, D. C.; Kim, B. J.; J. Ind. Eng. Chem. 2017, $54,324$.

35. Li, M.; Liu, H.; Chen, T.; Chen, D.; Wang, C.; Wei, L.; Wang, L.; Sci. Total Environ. 2020, 703, 135604.

36. Costa, R. C. C.; Lelis, M. F. F.; Oliveira, L. C. A.; Fabris, J. D.; Ardisson, J. D.; Rios, R. R. V. A.; Silva, C. N.; Lago, R. M.; J. Hazard. Mater. 2006, 129, 171.

37. Verma, M.; Haritash, A. K.; J. Environ. Chem. Eng. 2019, 7, 102886.

38. da Silva, L. A.; Borges, S. M. S.; Paulino, P. N.; Fraga, M. A.; Oliva, S. T.; Marchetti, S. G.; Rangel, M. C.; Catal. Today 2017, 289, 237.

39. Ren, B.; Xu, Y.; Zhang, C.; Zhang, L.; Zhao, J.; Liu, Z.; J. Taiwan Inst. Chem. Eng. 2019, 97, 170.

40. Masset, P.; Poinso, J.; Poignet, J.; J. Therm. Anal. Calorim. 2006, 83, 457.

41. Zhang, X.; Li, Y.; Li, G.; Hu, C.; RSC Adv. 2015, 5, 4984.

42. Honorato, A. C.; Machado, J. M.; Celante, G.; Borges, W. G. P.; Dragunski, D. C.; Caetano, J.; Rev. Bras. Eng. Agríc. Ambient. 2015, 19, 705 .

43. Augusto, T. d. M.; Chagas, P.; Sangiorge, D. L.; Mac Leod, T. C. d. O.; Oliveira, L. C. A.; Castro, C. S.; J. Environ. Chem. Eng. 2018, 6, 6545.

44. Rahim Pouran, S.; Abdul Raman, A. A.; Wan Daud, W. M. A.; J. Cleaner Prod. 2014, 64, 24.

45. Shi, X.; Li, X.; Liu, G.; Du, M.; Zhang, J.; Liu, G.; Lu, Q.; J. Mater. Sci. 2020, 55, 10035.

46. Wang, Q.; Tian, S.; Ning, P.; Ind. Eng. Chem. Res. 2014, 53, 643.

47. Liang, X.; Zhong, Y.; Zhu, S.; Ma, L.; Yuan, P.; Zhu, J.; He, H. Jiang, Z.; J. Hazard. Mater. 2012, 199, 247.

48. Sheldon, R.A; Wallau, M.; Arends, I.; Schuchardt, U.; Acc. Chem. Res. 1998, 31, 485.

49. Mantovani, M.; Aguiar, E. M.; Carvalho, W. A.; Mandelli, D.; Gonçalves, M.; Quim. Nova 2015, 38, 526. 